S Research S Suare

\title{
A study on orderings based on fuzzy quasi- order relations
}

\section{Zhonglin Chai ( $\square$ chaizhonglin@163.com )}

China Jiliang University https://orcid.org/0000-0003-4675-7589

\section{Research Article}

Keywords: Fuzzy quasi-order relation, Ordering, Partial order relation, Fuzzy graph

Posted Date: May 3rd, 2021

DOI: https://doi.org/10.21203/rs.3.rs-268904/v1

License: (a) (i) This work is licensed under a Creative Commons Attribution 4.0 International License. Read Full License 


\title{
A study on orderings based on fuzzy quasi-order relations
}

\author{
Zhonglin Chai ${ }^{\mathrm{a}, \mathrm{b}}$ \\ ${ }^{a}$ College of Sciences, ${ }^{b}$ Key Laboratory of Intelligent Manufacturing Quality Big Data Tracing and Analysis \\ of Zhejiang Province, China Jiliang University, Hangzhou 310018, China
}

\begin{abstract}
This paper further studies orderings based on fuzzy quasi-order relations using fuzzy graph. Firstly, a fuzzy relation on a finite set is represented equivalently by a fuzzy graph. Using the graph, some new results on fuzzy relations are derived. In ranking those alternatives, we usually obtain a quasi-order relation, which often has inconsistencies, so it cannot be used for orderings directly. We need to remake it into a reasonable partial order relation for orderings. This paper studies these inconsistencies, and divides them into two types: framework inconsistencies and degree inconsistencies. For the former, a reasonable and feasible method is presented to eliminate them. To eliminate the latter, the concept of complete partial order relation is presented, which is more suitable than partial order relation to rank the alternatives. A method to obtain a reasonable complete partial order relation for a quasi-order relation is given also. An example is given as well to illustrate these discussions. Lastly, the paper discusses the connection between quasi-order relations and preference relations for orderings and some other related problems.
\end{abstract}

Keywords: Fuzzy quasi-order relation; Ordering; Partial order relation; Fuzzy graph

\section{Introduction}

In the real world, many things have connections or relationships between them, so we need to study them(Rosen 2008). When the connections have uncertain or fuzzy trait, we should use fuzzy theories to study them(Bodenhofera and Klawonn 2004). Fuzzy relations are a generalization of crisp relations, and form one of the primary substances of fuzzy theory. Zadeh is the pioneer of fuzzy theory, and the first man who studied fuzzy relations (Zadeh 1965,1971). Since then, a lot of scholars have joined the queue of studying fuzzy relations (González and Marín 1997;Kundu 2000; Tan 2013;Zhang et al. 2020).

Relations and graphs both belong to discrete mathematics, possessing many common characteristics, so graphs can be used to study relations (Rosen 2008). Studying fuzzy relations needs fuzzy graphs, which were introduced by Rosenfeld (1975), and a lot of people have then interested in them (Bhutani and Rosenfeld 2003; Mathew and Sunitha 2009; Sameena and Sunitha 2006). Many results on fuzzy graphs have been gotten, and they have been used in many domains (Peng and Sun 2007;Hu 2014;Binu et al. 2019) . Naturally, some scholars have used graphs to study fuzzy relations (Imai et al. 2000;Kundu 2000;Mathew and Sunitha 2010). Although as it is, some connections between fuzzy graphs and relations still need to study.

In fuzzy relations, partial order relations are an important content of study as they can be used to rank alternatives(Kundu 2000;Bodenhofera and Klawonn 2004;Peng and Sun 2007). In practical problems, however, the relation obtained often is a quasi-order relation rather than a partial order relation(Liang et al. 2005; Ma et al. 2006), so how to use the relation to obtain a reasonable partial order relation for orderings has long been a problem. Besides, many scholars have focused their interest on studying preference relations and using them for orderings (Viedma et al. 2004; Xu et al. 2013;Zhang 2019). 
The paper aims to use fuzzy graph to study fuzzy quasi-order relations, and then use the derived results to study orderings based on them. The paper is organized as follows. Section 2 reviews some basic definitions and results of fuzzy relations. Section 3 gives some basic definitions and results of fuzzy graphs. The graphic representation of fuzzy relations is also given. Section 4 studies fuzzy quasi-order relations using fuzzy graph. Section 5 studies how to obtain a reasonable fuzzy partial order relation for ordering by a quasi-order relation. Firstly, it gives a method to obtain a reasonable partially ordered fuzzy quasi-order relation for a non-partially ordered quasi-order relation. Second, it gives a method to obtain a reasonable fuzzy partial order relation for a partially ordered quasi-order relation. An example is given as well to show the studies. Lastly, some discussions about the connection between quasi-order relations and preference relations for orderings and some other related problems are given.

\section{Definitions and some results on fuzzy relations}

In this paper, $X$ always denotes the finite set of alternatives $\left\{x_{1}, x_{2}, \ldots, x_{n}\right\}(n \geq 2)$, where $x_{i}$ denotes the $i$ th alternative; moreover, any relation is fuzzy in the following.

Let $R$ be a relation on $X$, which can be represented by a fuzzy matrix $R=\left(r_{i j}\right)_{n \times n} \in F(X \times X)$ (simply by $\left(r_{i j}\right)$ ). Thus, some well known definitions and results about relations are given according to fuzzy matrices.

Definition 2.1(Zadeh $1971 ;$ Hu 2014). Let $R=\left(r_{i j}\right)$ be a fuzzy relation on $X, R$ is called

(1) Reflexive iff $r_{i i}=1$ for any $i$,

(2) Symmetric iff $r_{i j}=r_{j i}$ for any $i, j$,

(3) Antisymmetric iff for any $i \neq j$ there is $r_{j i}=0$ when $r_{i j}>0$,

(4) Transitive iff $R^{2} \subseteq R$.

Notice: here the operators in computing $R^{2}$ are the two famous operators $\wedge$ and $\vee$.

Definition 2.2(Zadeh 1971; Hu 2014). Let $R$ be a fuzzy relation on $X$,

(1) $R$ is a quasi-order relation iff it is reflexive and antisymmetric,

(2) $R$ is a partial order relation iff it is reflexive, antisymmetric, and transitive.

Definition 2.3(Zadeh 1971; Hu 2014). Let $R$ be a fuzzy relation on $X$, a relation $A$ containing $R$ is transitive, and for any relation $B$ containing $R$ with transitivity there always is $B \supseteq A$, then $A$ is called the transitive closure of $R$, denoted by $R^{t}$.

Theorem 2.1(Imai et al. 2000;Peng and Sun 2007;Hu 2014). Let $R$ be a fuzzy relation on $X$ with reflexivity, then

(1) $R^{k}$ is reflexive for any positive integer $k$,

(2) There is $R^{s} \subseteq R^{k}$ for any two positive integers $s \leq k$,

(3) There exists an integer $k \leq n$-1 such that $R^{k}=R^{k+1}=\ldots=R^{t}$.

From Definitions 2.1, 2.2 and Theorem 2.1 there is

Corollary 2.1. A sufficient and necessary condition that a quasi-order relation $R$ on $X$ is partial order is that $R=R^{2}$.

Let $R$ be a quasi-order relation. If $R^{t}$ still is antisymmetric, $R^{t}$ is a partial order relation by Definitions 2.1, 2.2 and Theorem 2.1, so

Definition 2.4(Peng and Sun 2007; Hu 2014). Let $R$ be a fuzzy quasi-order relation on $X$, if $R^{t}$ still is antisymmetric, $R$ is called partially ordered.

Notice: As this paper mainly discusses order relations for the given matrix $R=\left(r_{i j}\right)$, so we need 
further give some explanations about it. Suppose $r_{i j}(i \neq j)$ denotes the degree of $x_{i}$ superior to $x_{j}$ when comparing them, i.e., $r_{i j}=0$ when $x_{i}$ is not superior (or is inferior) to $x_{j}$ or they can not be compared, $r_{i j}>0$ when $x_{i}$ is superior to $x_{j}$ (denoted by $x_{i}>x_{j}$ ), and the greater the value, the greater the degree, and $r_{i j}=1$ means $x_{i}$ is completely superior to $x_{j}$.

\section{Definitions and some results of fuzzy graphs}

In this section, we give the graphic representation of fuzzy relations and review some definitions of fuzzy graphs.

Definition 3.1(Peng and Sun 2007; Rosen 2008; Ghosh et al. 2010). A fuzzy graph is a weighted graph, which can be undirected or directed, but the weight must be in $(0,1]$.

For a fuzzy relation $R=\left(r_{i j}\right)$ on $X$, if some $r_{i j}$ are different from $r_{j i}$, it can be represented equivalently by a directed graph, where $X$ is the vertex set. If $r_{i j} \neq 0$, an arrow is used pointing from $x_{i}$ to $x_{j}$ to indicate the directed edge from $x_{i}$ to $x_{j}$, and $r_{i j}$ is the weight. If $r_{i j}=0$, such an edge does not exist.

For two vertices $x_{i}$ and $x_{j}$ of the graph of a relation $R=\left(r_{i j}\right)$, if $x_{i}$ is adjacent to $x_{j}$, the edge is denoted by $\left(x_{i}, x_{j}\right)$. Sometimes $\left(x_{i}, x_{j}\right)$ is represented by $r_{i j}$ when there is no danger of confusion.

When $R$ is symmetric, we need not differentiate between the two edges $r_{i j}$ and $r_{j i}$, so $R$ can be represented equivalently by an undirected graph, where $X$ is the vertex set, $x_{i}$ is adjacent $x_{j}$ when $r_{i j}>0$ or $r_{j i}>0$, and the edge is denoted by $\left\{x_{i}, x_{j}\right\}$ or $\left\{x_{j}, x_{i}\right\}$.

Conversely, a fuzzy graph $G$ can be represented equivalently by the form $(X, R)$ (here we do not consider the situation of multi-graphs), where $X$ is the vertex set, $R$ is the relation derived from $G$. If $G$ is directed, the $R$ is undoubted by the graphic representation of fuzzy relations. If $G$ is undirected, we use a symmetric matrix to represent the relation, so the $R$ is also clear. Thus in the following, we always use $G=(X, R)$ (sometimes $G$ is used only) to represent the fuzzy graph (simply called graph). Besides, as this paper mainly discusses order relations, so any graph is directed in the following.

For a relation $R$, its graph $G$ is called the graph of $R$, denoted by $G_{R}$; for a graph $G=(X, R), R$ is called the relation of graph $G$, denoted by $R_{G}$.

Definition 3.2(Rosen 2008; Mathew and Sunitha 2009; Ghosh et al. 2010). Let $G=(X, R)$ be a fuzzy graph, the vertex sequence $x_{0}, x_{1}, \ldots, x_{k}(k>0)$, in which $x_{i-1}$ is adjacent to $x_{i}(i=1,2, \ldots, k)$, is called a walk, where $x_{0}$ and $x_{k}$ are called the initial vertex and terminal vertex of the walk, respectively, $k$ is called the length of the walk.

Definition 3.3(Rosen 2008; Mathew and Sunitha 2009; Ghosh et al. 2010). Let $G=(X, R)$ be a fuzzy graph, $x_{0}, x_{1}, \ldots, x_{k}$ a walk in $G$, if the $x_{i}$ are distinct ( $x_{0}$ and $x_{k}$ are possible the same), it is called a path from $x_{0}$ to $x_{k}$. If $x_{0}$ and $x_{k}$ are the same and $k=1$, it is called a loop; if $x_{0}$ and $x_{k}$ are the same and $k \geq 2$, it is called a closed path or cycle.

Definition 3.4(Peng and Sun 2007; Mathew and Sunitha 2009;Hu 2014). Let $G=(X, R)$ be a fuzzy graph, $x_{0}, x_{1}, \ldots, x_{k}$ a walk $W$ in $G$, denote $S(W)=\wedge_{i=1}^{k} r_{i-1 i}, \mathrm{~S}(W)$ is called the strength of $W$.

Definition 3.5(Peng and Sun 2007; Hu 2014). Let $G=(X, R)$ be a fuzzy graph, $x_{i}, x_{j} \in X, P_{1}, P_{2}, \ldots$, $P_{l}$ are all paths in $G$ from $x_{i}$ to $x_{j}$, denote $S_{i j}=\stackrel{l}{\vee} S\left(P_{i}\right), S_{i j}$ is called the connectivity strength from $x_{i}$ to $x_{j}$ in $G$. 
Definition 3.6(Peng and Sun 2007; Hu 2014). Let $G=(X, R)$ be a fuzzy graph, $P$ a path from $x_{i}$ to $x_{j}$, if $\mathrm{S}(P)=S_{i j}$, $\mathrm{P}$ is called a strong path from $x_{i}$ to $x_{j}$ in $G$.

Besides, quasi-order relations are reflexive, indicating that their graphs have a loop at each vertex weighting 1 .

\section{Study fuzzy relations using fuzzy graph}

Now we use fuzzy graphs to study quasi-order relations.

Theorem 4.1. A sufficient and necessary condition that fuzzy quasi-order relation $R=\left(r_{i j}\right)$ on $X$ is partially ordered is that $G_{R}$ has no cycles.

Proof. As a cycle has at least two different vertices from Definition 3.3, so we only consider any two different alternatives $x_{i}, x_{j}$.

Sufficiency. Suppose there is a positive integer $k \leq n-1$ such that $R^{k}=R^{t}$ from Theorem 2.1(3). Let $R^{k}=\left(r^{k} i j\right)$, then there is (Zadeh 1965;Peng and Sun 2007)

$$
r^{k}{ }_{i j}=\underset{j_{1}, j_{2}, \ldots j_{k-1}}{\vee}\left(r_{i j_{1}} \wedge r_{j_{1} j_{2}} \wedge \ldots \wedge r_{j_{k-1} j}\right)
$$

From Definition 2.1(3) if $r_{j i}>0$, then $r_{i j}=0$. From Theorem 2.1(2) there still is $r_{j i}^{k}>0$. If $r_{i j}^{k}>0$, then by (4.1) there are edges $r_{i j_{1}}, r_{j_{1} j_{2}}, \ldots, r_{j_{k-1} j}$ such that $r_{i j_{1}}>0, r_{j_{1} j_{2}}>0, \ldots, r_{j_{k-1} j}>0$. These edges together with $r_{j i}$ form a closed walk, so a cycle exists, a contradiction, thus $r_{i j}^{k}=0$. If $r_{i j}=r_{j i}=0$, we consider $r_{i j}^{k}$ and $r_{j i}^{k}$, by a similar way we see that they cannot be both greater than 0 . These show that $R^{t}$ still is antisymmetric, hence $R$ is partially ordered from definition 2.4.

Necessity. Suppose $R$ is partially ordered. If $G_{R}$ had a cycle, in which the edges are $r_{i j_{1}}, r_{j_{1} j_{2}}, \ldots, r_{j_{k} i}\left(1 \leq k \leq n-1\right.$ by Definition 3.3), we consider $R^{k}$. From Theorem 2.1(2), (4.1), and $k \leq n-1$ there are $r^{k}{ }_{i j_{1}}>0, r_{j_{1} i}^{k}>0$, so $r^{n-1}{ }_{i j_{1}}>0, r_{j_{1} i}^{n-}>0$. This shows that $R^{t}$ is not antisymmetric from Theorem 2.1(3), a contradiction. This completes the proof.

Theorem 4.2. A sufficient and necessary condition that quasi-order relation $R=\left(r_{i j}\right)$ on $X$ is partial order is that if there are paths from $x_{i}$ to $x_{j}$ in $G_{R}$, then $x_{i}$ is adjacent to $x_{j}$, and the edge $\left(x_{i}, x_{j}\right)$ is a strong path from $x_{i}$ to $x_{j}$ in $G_{R}$.

Proof. Obviously, we only need to consider the case that $x_{i} \neq x_{j}$.

Sufficiency. For any $x_{i}$ and $x_{j}$, if there are no paths from $x_{i}$ to $x_{j}$, then there are $r_{i j}=0$ and $\underset{k=1}{\vee}\left(r_{i k} \wedge r_{k j}\right)=0$. If there are paths from $x_{i}$ to $x_{j}$, as the edge $\left(x_{i}, x_{j}\right)$ is a strong path, then for any $k$, there is $r_{i j} \geq r_{i k} \wedge r_{k j}$ from Definitions 3.4-3.6. Hence for both cases there is $r_{i j} \geq \underset{k=1}{\vee}\left(r_{i k} \wedge r_{k j}\right)$, thus $R^{2} \subseteq R$. So $R$ is partial order from Definitions 2.1, 2.2.

Necessity. If $R$ is partial order, from Corollary 2.1 there is $R=R^{n-1}$, i.e.,

$$
r_{i j}=\underset{j_{1}, j_{2}, \ldots, j_{n-2}}{\vee}\left(r_{i j_{1}} \wedge r_{j_{1} j_{2}} \wedge \ldots \wedge r_{j_{n-2} j}\right)
$$

For any $x_{i}$ and $x_{j}$, if there are no paths from $x_{i}$ to $x_{j}$, then $r_{i j}=0$ by (4.2) obviously. Now consider the case that there are paths from $x_{i}$ to $x_{j}$. For a path in $G_{R}$, the connectivity strength of a walk which is obtained from it by adding some vertices through loops is unchanged. So for a path, there 
is a walk $r_{i j_{1}} \wedge r_{j_{1} j_{2}} \wedge \ldots \wedge r_{j_{n-2} j}$ whose strength is equal to that of the path. Thus, $r_{i j}>0$ from (4.2). One sees that the strength of any path is not greater than $r_{i j}$, so $x_{i}$ is adjacent to $x_{j}$, and the edge $\left(x_{i}\right.$, $x_{j}$ ) is a strong path from $x_{i}$ to $x_{j}$. This completes the proof.

\section{Find a reasonable partial order relation for a quasi-order relation}

In real problems, we often need to rank some alternatives, so partial order relations are needed as they can be used for orderings. When we are deriving such a relation, however, due to the availability and uncertainty of information as well as the vagueness of human feeling and recognition, the entry in the obtained relation often is inaccurate or approximate (Liang et al. 2005;Ma et al. 2006). Thus, we need a partial order relation but the harvest often is a quasi-order relation. Moreover, in the relation, the phenomenon of inconsistencies may appear. Ma et al.(2006) and $\mathrm{Xu}$ et al. (2013) discussed it mainly for the case that $r_{i j}>0, r_{j k}>0$, and $r_{k i}>0$. In fact it has no essential difference with the case that $r_{i j}>0$ and $r_{j i}>0$, and that $r_{i j}>0, r_{j k}>0, r_{k s}>0$, and $r_{s i}>0$ is only a more complex case. In real problems, the case that $r_{i j}>0$ and $r_{j i}>0$ can be avoided, but the phenomenon of inconsistencies is difficult to avoid. Inconsistency is a contradiction phenomenon among the alternatives. Now, let's discuss it.

Definition 5.1. For quasi-order relation $R=\left(r_{i j}\right)$ on $X$, if there are some different alternatives $x_{i}$ of $X$ such that $r_{i j_{1}}>0, r_{j_{1} j_{2}}>0, \ldots, r_{j_{k} i}>0(k>0), R$ is called inconsistency.

How to test that a quasi-order relation $R$ is inconsistency? From Definition 5.1 when $R$ is inconsistency, cycles in $G_{R}$ exist, so from Theorem 4.1 there is

Theorem 5.1. A sufficiency and necessary condition that quasi-order relation $R=\left(r_{i j}\right)$ on $X$ is inconsistent is that its graph $G_{R}$ has cycles, it is non-partially ordered, or its transitive closure $R^{t}$ is not antisymmetric.

In orderings by quasi-order relation $R=\left(r_{i j}\right)$ on $X$, a common used method(Peng and Sun $2007 ; \mathrm{Hu} 2014$ ) is computing its transitive closure $R^{t}$ firstly. If it still is antisymmetric, it is partial order, so it can be used for orderings. Otherwise, use a new relation, denoted by $A=\left(a_{i j}\right)$, where

$$
a_{i j}=\left\{\begin{array}{c}
r_{i j}^{t}-r_{j i}^{t}, \text { if } i \neq j, r_{i j}^{t}>r^{t}{ }_{j i} \\
0, \text { if } i \neq j, r^{t}{ }_{i j} \leq r^{t}{ }_{j i} \\
r^{t}{ }_{i j}, \quad \text { if } i=j
\end{array}\right.
$$

It is a partial order relation, can be used for orderings.

For a non-partially ordered quasi-order relation $R=\left(r_{i j}\right)$ on $X$, i.e., its $G_{R}$ has cycles, we can convert it into a partially ordered quasi-order relation by cutting some edges for each cycle, i.e., let the corresponding $r_{i j}$ be 0 . Obviously, the manner of preserving the original information of $R$ as much as possible is cutting edges as little as possible, and the most reasonable manner of cutting edges is cutting the edge having the least weight among the edges for each cycle. Thus, the best manner of converting a quasi-order relation with inconsistencies into a quasi-order relation with consistency is cutting only one edge having the least weight for each cycle. Besides, there is another manner to eliminate those inconsistencies: let the weights of all the edges in a cycle decrease by the number of the smallest weight of those weights (so the edge having the smallest weight is cut naturally). For these two ways, the latter is poorer as the loss of the original 
information of $R$ for the former is less than that of the latter.

Now we consider the former way:cut an edge having the smallest weight for each cycle in the graph of $R$. But how to know that an edge in a cycle is the one having the smallest weight to the cycle? For this, we have the following theorems.

Theorem 5.2. Let $R=\left(r_{i j}\right)$ be a fuzzy quasi-order relation on $X$ whose graph $G_{R}$ has only one cycle. Then for the edge $r_{i j}(>0)$ in the cycle having the smallest weight to the cycle, there is $r_{i j}^{t} \leq r_{j i}^{t}$, and for any other edges $r_{i j}$ in $G_{R}$, there are $r_{i j}^{t}>r_{j i}^{t}$.

Proof. Consider an edge $r_{i j}(>0)$ in $G_{R}$. From Theorem 2.1(2)(3) there is $r_{i j}^{t}>0$. Suppose $r_{i j}$ is not in the cycle. If $r_{j i}^{t}>0$,then there will be a path from $x_{j}$ to $x_{i}$ from (4.1). This path together with $r_{i j}$ forms a cycle. It is a new cycle, a contradiction, so there is $r_{j i}^{t}=0$, then $r_{i j}^{t}>r_{j i}^{t}$.

Let this cycle be $x_{0}, x_{1}, \ldots, x_{k}\left(x_{0}=x_{k}, k>2\right)$,consider $r_{i j}$ is in it. First consider that $r_{i j}$ has the smallest weight to it, say $r_{01}$. Consider the path from $x_{0}$ to $x_{1}$. Obviously, $r_{01}$ is a path. If there is another path from $x_{0}$ to $x_{1}$, then this path together with $x_{1}, \ldots, x_{k}$ forms a new cycle, a contradiction. This shows that $r_{01}$ is the only path from $x_{0}$ to $x_{1}$, so $r_{01}^{t}=r_{01}$ by the proof of Theorem 4.2. Now consider $r^{t}$. Obviously, $x_{1}, \ldots, x_{k}$ is a path from $x_{1}$ to $x_{0}$, one sees that the strength of this path is not less than $r_{01}$, so $r_{10}^{t} \geq r_{01}=r_{01}^{t}$ by the proof of Theorem 4.2.

Now consider that $r_{i j}$ is in the cycle not having the smallest weight,still suppose that it is $r_{01}$. Suppose that the smallest weight of edges in $x_{0}, x_{1}, \ldots, x_{k}$ is $a$, then $r_{01}>a$. There is $r_{01}^{t}>a$ obviously. Consider $r^{t} 10$. By a similar analysis as above we know that $x_{1}, \ldots, x_{k}$ is the only path from $x_{1}$ to $x_{0}$, and the edge with the smallest weight in the cycle is in this path, so $r^{t}{ }_{10}=a<r^{t}{ }_{01}$. This completes the proof. $\square$

For quasi-order relation $R=\left(r_{i j}\right)$, when $G_{R}$ has many cycles but any two of them do not intersect, the conclusions of Theorem 5.2 still hold obviously. We can use $r_{i j}>0$ and $r_{i j}^{t} \leq r_{j i}^{t}$ to select the edge having the smallest weight in the cycle. When $G_{R}$ has many cycles and at least two cycles intersect, we have the following theorem.

Theorem 5.3. Let $R=\left(r_{i j}\right)$ be a fuzzy quasi-order relation on $X$ whose graph $G_{R}$ has cycles, then there is at least one edge in the cycles such that $r_{i j}>0$ and $r_{i j}^{t} \leq r_{j i}^{t}$.

Proof. Consider a cycle $x_{0}, x_{1}, \ldots, x_{k}\left(x_{0}=x_{k}, k>2\right)$ of $G_{R}$. Obviously for each edge $r_{i j}$ in it, there is $r_{i j}>0$. By the proof of Theorem 5.2 there are $r_{i j}^{t}>0$ and $r_{j i}^{t}>0$. For these $r_{i j}^{t}$, they have their minimum, say $r_{01}^{t}$. Of course, the transitive closure of $R^{t}$ still is $R^{t}$. This shows that for any $r_{i j}$ in the cycle, $r_{i j}^{t}$ is a strong path in $G_{R}{ }^{t}$, the graph of $R^{t}$, from $x_{i}$ to $x_{j}$ by (4.1). As $r^{t} 01$ is the minimum of these $r_{i j}^{t}$, and they form a cycle, there must be $r^{t}{ }_{10} \geq r_{01}^{t}$ by the proof of Theorem 5.2. Obviously $r_{01}>0$, and this completes the proof.

Based on Theorems 5.2, 5.3, we give an algorithm to obtain a reasonable partially ordered quasi-order relation for a non-partially ordered quasi-order relation.

\section{Algorithm 1.}

Step 1. For quasi-order relation $R=\left(r_{i j}\right)$, compute $R^{t}=\left(r_{i j}^{t}\right)$.

Step 2. If $R^{t}$ is antisymmetric, stop. Otherwise, go to the next step.

Step 3. If $r_{i j}$ satisfies $r_{i j}>0$, and $r_{i j}^{t} \leq r_{j i}^{t}$, and it is the edge whose weight has the smallest value among those edges having these properties, replace it with 0 (the obtained relation is still denoted by $R$ for convenience), then go to Step 1 .

Now we see an example.

Example 1. Let $X=\left\{x_{1}, \quad x_{2}, \quad x_{3}, x_{4}, x_{5}\right\}$,consider quasi-order relation 
$\begin{aligned} R & =\left(\begin{array}{lllll}1 & .7 & .3 & .37 & .49 \\ 0 & 1 & 0 & .05 & 0 \\ 0 & .3 & 1 & 0 & 0 \\ 0 & 0 & .16 & 1 & 0 \\ 0 & .6 & .36 & .45 & 1\end{array}\right) \text { on } X \text {. The transitive closure of } R \text { is } \\ R^{t} & =\left(\begin{array}{lllll}1 & .7 & .36 & .45 & .49 \\ 0 & 1 & .05 & .05 & 0 \\ 0 & .3 & 1 & .05 & 0 \\ 0 & .16 & .16 & 1 & 0 \\ 0 & .6 & .36 & .45 & 1\end{array}\right) . R^{t} \text { is not antisymmetric, showing there are cycles in } G_{R} \text {. From }\end{aligned}$

$R^{t}$ for the three elements $x_{2}, x_{3}, x_{4}$, there are $r_{i j}^{t}>0$ and $r_{j i}^{t}>0$, but it is not for $x_{1}, x_{5}$. So by the proof of Theorem 5.2 we know that $x_{1}, x_{5}$ are not in the cycle, but $x_{2}, x_{3}, x_{4}$ are in(see Fig.1). In fact for this example, the cycle is unique. From $R$ the cycle is formed by the three edges $r_{24}, r_{43}$ and $r_{32}$. For the three edges,only $r_{24}$ satisfies $r_{i j}>0$ and $r_{i j}^{t} \leq r_{j i}^{t}$, so $r_{24}$ is the unique edge with the smallest weight in the cycle. Cut it, i.e., replace $r_{24}$ with 0 , and still denote the new relation by $R$. Now, $R=\left(\begin{array}{ccccc}1 & .7 & .3 & .37 & .49 \\ 0 & 1 & 0 & 0 & 0 \\ 0 & .3 & 1 & 0 & 0 \\ 0 & 0 & .16 & 1 & 0 \\ 0 & .6 & .36 & .45 & 1\end{array}\right)$, and it is easy to know it is partially ordered.

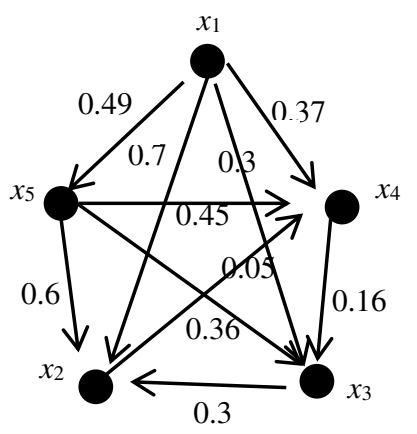

Fig. 1. Graph of $R$ of Example 1

When quasi-order relation $R=\left(r_{i j}\right)$ on $X$ has inconsistencies, i.e., its graph has cycles, for any two distinct $x_{i}$ and $x_{j}$ in a cycle of $G_{R}$, there are $r_{i j}^{t}>0$ and $r_{j i}^{t}>0$, so all these $r_{i j}^{t}$ will be changed when using (5.1). Thus the method of (5.1) is just similar to the above latter way to eliminate the inconsistency, so the original information of $R$ is changed greatly.

Consider this method and Example 1 again, by (5.1) the obtained relation is 
$\begin{aligned} A & =\left(\begin{array}{ccccc}1 & .7 & .36 & .45 & .49 \\ 0 & 1 & 0 & 0 & 0 \\ 0 & .25 & 1 & 0 & 0 \\ 0 & .11 & .11 & 1 & 0 \\ 0 & .6 & .36 & .45 & 1\end{array}\right) \text {. It is the transitive closure of } \\ R_{1} & =\left(\begin{array}{ccccc}1 & .7 & .3 & .37 & .49 \\ 0 & 1 & 0 & 0 & 0 \\ 0 & .25 & 1 & 0 & 0 \\ 0 & 0 & .11 & 1 & 0 \\ 0 & .6 & .36 & .45 & 1\end{array}\right) \text {. Compared with the initial } R, \text { the } 0.05 \text { is converted into } 0 \text {, at }\end{aligned}$ the same time 0.3 and 0.16 are converted into 0.25 and 0.11 respectively. Obviously, it is unreasonable.

Now we only consider partially ordered quasi-order relations. As given in Sec.2, we use $r_{i j}$ $(i \neq j)$ to denote the degree of $x_{i}$ superior to $x_{j}$. Obviously from the point of logic, for three different elements $x_{i}, x_{j}, x_{k}$, if $x_{i}$ is superior to $x_{j}$ and $x_{j}$ is superior to $x_{k}$, then $x_{i}$ should be more superior to $x_{j}$. That is, if $r_{i j}>0$ and $r_{j k}>0$, then there should be $r_{i k} \geq r_{i j}$ and $r_{i k} \geq r_{j k}$, i.e., $r_{i k} \geq r_{i j} \vee r_{j k}$. Consider it generally, we give

Definition 5.2. Let $R=\left(r_{i j}\right)$ be a fuzzy partial order relation on $X$, if for any path $x_{1}, x_{2}, \ldots, x_{k}$ of $G_{R}$, there always is $r_{1 k} \geq \underset{i=2}{\vee} r_{i-1 i}, R$ is called a complete fuzzy partial order relation.

Example 1 (continued).

Consider Example1 continuously and the last $R$. As $r_{42}=0$, but $r_{43}=0.16, r_{32}=0.3$, so $R$ is not a complete partial order relation. Even we consider its transitive closure $R^{t}=\left(\begin{array}{ccccc}1 & .7 & .36 & .45 & .49 \\ 0 & 1 & 0 & 0 & 0 \\ 0 & .3 & 1 & 0 & 0 \\ 0 & .16 & .16 & 1 & 0 \\ 0 & .6 & .36 & .45 & 1\end{array}\right)$, which is partial order, it still is not complete partial order as $r_{13}=0.36$, but $r_{15}=0.49, r_{53}=0.36$. This means we can not obtained complete partial order relations by transitive closure method.

The inconsistency defined by Definition 5.1 for quasi-order relation $R=\left(r_{i j}\right)$ on $X$ should be called framework inconsistency as it reflects there are framework or order contradictions among some elements of $X$, and the inconsistency here should be called degree inconsistency as it reflects there are degree contradictions among some elements of $X$. A partial order relation on $X$ means there are no framework contradictions on the elements of $X$. Although it can be used for orderings, it does not mean there are not degree contradictions among the elements of $X$. However, it is not for complete partial order relations. So for a quasi-order relation, we should find a reasonable complete partial order relation to show the order relations of the elements of $X$. To complete this work, we need some preparations.

Definition 5.3. Let $G=(X, R)$ be a fuzzy graph, $x_{0}, x_{1}, \ldots, x_{k}$ a path $P$ in $G$, denote 
$S S(\mathrm{P})=\stackrel{k}{\vee} r_{i=1}, S S(P)$ is called the strong strength of $P$.

Definition 5.4. Let $G=(X, R)$ be a fuzzy graph, $x_{i}, x_{j} \in X, P_{1}, P_{2}, \ldots, P_{l}$ are all paths in $G_{R}$ from $x_{i}$ to $x_{j}$, denote $S S_{i j}=\stackrel{l}{\vee} S S\left(P_{i}\right), S S_{i j}$ is called the strong connectivity strength in $G$ from $x_{i}$ to $x_{j}$. If there are no paths from $x_{i}$ to $x_{j}, S S_{i j}=0$.

Theorem 5.4. Let $R=\left(r_{i j}\right)$ be a fuzzy partially ordered quasi-order relation on $X, R^{s}=\left(s_{i j}\right)$ a fuzzy relation on $X$ in which each $s_{i j}$ is determined by the $S S_{i j}$ of $R$, then

(1) $R^{s}=\left(s_{i j}\right)$ is a complete partial order relation;

(2) It is the least complete partial order relation among complete partial order relations on $X$ containing $R=\left(r_{i j}\right)$.

Proof. (1) For any $i$, as $r_{i i}=1$ and loops belong to paths, so there is $s_{i i}=1$ by Definitions 5.3,5.4. Consider $i \neq j$. If $s_{i j}>0$, then $s_{j i}=0$, otherwise there will exist a contradiction with $R$ being partially ordered or its graph having no cycles by the proof of Theorem 4.1, so $R^{s}$ is quasi-order. Consider $s_{i j}>0$, suppose there are a path $\mathrm{P}$ of the graph of $R^{s}$ from $x_{i}$ to $x_{j}$ and an edge $\left(x_{k}, x_{s}\right)$ belonging to $\mathrm{P}$ such that $s_{k s}>s_{i j}$, by Definitions 5.3,5.4 there will exist a path $\mathrm{P}_{1}$ of the graph of $R$ from $x_{k}$ to $x_{s}$ and a path $\mathrm{P}_{2}$ from $x_{i}$ to $x_{j}$ and the former is a part of the latter such that $S S\left(\mathrm{P}_{1}\right)>S S\left(\mathrm{P}_{2}\right)$, a contradiction with Definition 5.3. Thus, for any path $\mathrm{P}$ of the graph of $R^{s}$ from $x_{i}$ to $x_{j}$ and any edge $\left(x_{k}, x_{s}\right)$ belonging to $\mathrm{P}$, there is $s_{k s} \leq s_{i j}$. Also consider the edge $s_{i j}$ itself, $R^{s}$ is partial order by the proof of Theorem 4.2. Besides, by Definition 5.2 and $s_{k s} s_{i j}$ one sees that $R^{s}$ is also complete.

(2) For any $i \neq j$, if $r_{i j}=0, s_{i j} \geq r_{i j}$ certainly. If $r_{i j}>0$, there still is $s_{i j} \geq r_{i j}$ by Definitions 5.3,5.4 as $r_{i j}$ is an edge of $G_{R}$, so $R^{s} \supseteq R$. Let $B=\left(b_{i j}\right)$ be a complete partial order relation on $X$ containing $R$, then there is $b_{i j} \geq r_{i j}$ for any $i, j$. By Definitions 5.2-5.4 there is $b_{i j} \geq s_{i j}$, so $B \supseteq R^{s}$. This completes the proof. $\square$

Thus for a quasi-order relation $R=\left(r_{i j}\right)$ on $X$, if it is non-partially ordered, we can use Algorithm 1 to let it become a partially ordered one. For a partially ordered quasi-order relation, we should use its $R^{s}$ for orderings. So the problem becomes for a partially ordered quasi-order relation $R=\left(r_{i j}\right)$ on $X$, how to find the $R^{s}$. We introduce the following operator $\theta$ : for any $a$, $b \in[0,1]$, define

$$
a \theta b=\left\{\begin{array}{c}
0, \quad \text { if } a \wedge b=0 \\
a \vee b, \quad \text { if } a \wedge b>0
\end{array}\right.
$$

Definition 5.5. Let $R=\left(r_{i j}\right)$ be a fuzzy relation on $X$, denote $R^{2(\theta)}=R^{\circ}{ }_{\theta} R=\left(r^{2(\theta)} i j\right)$, in which $R^{2(\theta)}$ is defined as follows: $r^{2(\theta)}{ }_{i j}=\underset{k=1}{\vee}\left(r_{i k} \theta r_{k j}\right)$, i.e., the composition is $\theta$ and $\vee$. Besides, let $R^{k(\theta)}=R^{\circ} R^{k-1(\theta)}, k>2$.

Let $R=\left(r_{i j}\right)$ be a partially ordered quasi-order relation on $X, R_{0}=R-I=\left(r_{0 i j}\right)$, in which $I$ is the identity matrix on $X$. Consider $R_{0}{ }^{k(\theta)}=\left(r_{0}{ }^{k(\theta)}{ }_{i j}\right), k>1$. By Definitions 5.3,5.4 and the proof of Theorem 4.1 one sees that $r_{0}{ }^{k(\theta)} i j$ is the maximum of strong strengths of all paths whose length is $k$ from $x_{i}$ to $x_{j}(i \neq j)$, thus $R^{s}$ can be obtained as follows $R^{s}=I \cup R_{0} \cup R_{0}^{2(\theta)} \cup \ldots \cup R_{0}^{n-1(\theta)}$.

Example 1 (continued).

Consider Example 1 continuously and the last $R$. By computation there is 
$R^{s}=\left(\begin{array}{ccccc}1 & .7 & .49 & .49 & .49 \\ 0 & 1 & 0 & 0 & 0 \\ 0 & .3 & 1 & 0 & 0 \\ 0 & .3 & .16 & 1 & 0 \\ 0 & .6 & .45 & .45 & 1\end{array}\right)$. It is the least complete partial order relation containing $R$,

suitable for orderings for the elements of $X$.

\section{Conclusions}

This paper further studies orderings based on quasi-order relations by fuzzy graph. For a given quasi-order relation come from reality, it often has inconsistencies, not only in the framework, but also in the numbers, so we need to obtain a reasonable partial order relation close to it for orderings. This paper studies how to eliminate these inconsistencies reasonably, presents methods to derive a reasonable partial order relation about the relation. The given example shows that the results are satisfactory. In pairwise comparisons, the relation given may not be the form of quasi-order relation $R=\left(r_{i j}\right)$, but the form of preference relation $A=\left(a_{i j}\right)$ which satisfies additive reciprocal: $a_{i j}+a_{j i}=1$ for any $i, j$ (Kundu 2000; Ma et al. 2006). But the two forms can be converted for one another by $r_{i j}=\left\{\begin{array}{c}2 a_{i j}-1, \text { if } i \neq j, a_{i j} \geq 0.5 \\ 0, \quad \text { if } i \neq j, a_{i j}<0.5 \\ 1, \quad \text { if } i=j,\end{array}\right.$ or $a_{i j}=\left\{\begin{array}{c}\left(r_{i j}+1\right) / 2, \text { if } i \neq j, r_{i j}>0 \\ 1-a_{j i}, \text { if } i \neq j, r_{j i}>0 \\ 0.5, \quad \text { if } i=j \text { or } r_{i j}=r_{j i},\end{array}\right.$. Thus, the orderings methods of this paper also work for preference relations. The given preference relation $A$ may satisfies multiplicative reciprocal: $a_{i j} \cdot a_{j i}=1$ for any $i, j$ (Viedma et al. 2004). But the two forms of reciprocal can be converted for one another(Viedma et al. 2004), so this paper's methods also work for this type of preference relations.

Besides, the $r_{i j}(i \neq j)$ in the given relation may not represent the degree of $x_{i}$ superior to $x_{j}$, but represent the degree of $x_{i}$ inferior to $x_{j}$. In this case above methods still work, we only need to see that each non-zero number in the last relation denotes the degree of the first element inferior to the second one.

In giving a quasi-order relation of the alternatives, the decision maker may be unable to give the values of some $r_{i j}$. If such numbers are rare, he can give them zero value, by transitivity operation or Theorem 5.4 for quasi-order relations these values can be given naturally, i.e., they can be determined by other given values $r_{i j}$. Certainly, sometimes the given quasi-order relation may be unreasonable, in such a case we should ask the decision maker to give a new one. But how to know it is? One sees when the graph of the given relation has cycles and we want to eliminate them by cutting some edges using the above method, if the value of one cut edge is great or not small, or such edges are too many, we can deem it is. Of course, we can change it into a preference relation and use the method for it to test(Xu and Wang 2013).

\section{Compliance with ethical standards}

Conflict of interest The author declares that he has no conflicts of interest.

Ethical approval This article does not contain any studies with human participants or animals performed by any of the authors. 


\section{References}

Bhutani KR, Rosenfeld A(2003) Strong arcs in fuzzy graphs. Inf Sci152:319-322

Binu M, Sunil M, Mordeson JN (2019) Connectivity index of a fuzzy graph and its application to human traffificking. Fuzzy Sets and Syst 360: 117-136

Bodenhofera U, Klawonn F(2004) A formal study of linearity axioms for fuzzy orderings. Fuzzy Sets and Syst 145: 323-354

Ghosh P, Kundu K, Sarkar D(2010) Fuzzy graph representation of a fuzzy concept lattice. Fuzzy Sets and Syst 161:1669 - 1675

González L, Marín A(1997) Weak properties and aggregated extension of fuzzy relations. Fuzzy Sets and Syst 85:311-318

Hu BQ(2014) Basis of fuzzy theory. Wuhan University Press, Wuhan (in Chinese)

Imai H, Okahara Y, Miyakoshi M(2000) The period of powers of a fuzzy matrix. Fuzzy Sets and Syst 109: 405-408

Kundu S(2000) Similarity relations, fuzzy linear orders, and fuzzy partial orders. Fuzzy Sets and Syst $109: 419-428$

Liang GS, Chou TY, Han TC(2005) Cluster analysis based on fuzzy equivalence relation. Eur. J.Oper. Res. 166:160-171

Ma J, Fan ZP, Jiang YP, Mao JY, Ma L(2006) A method for repairing the inconsistency of fuzzy preference relations. Fuzzy Sets and Syst 157: 20-33

Mathew S, Sunitha MS(2009) Types of arcs in a fuzzy graph. Inf Sci179:1760-1768

Mathew S, Sunitha MS(2010) Node connectivity and arc connectivity of a fuzzy graph. Inf. Sci. 180:519-531

Peng ZZ, Sun YY(2007) Fuzzy Mathematic and Its Application ( 2rd ed.). Wuhan University Press, Wuhan (in Chinese)

Rosen KH (2008) Discrete mathematics and its applications (6th ed.). China machine Press, Beijing

Rosenfeld A (1975) Fuzzy graphs, in: L.A. Zadeh, K.S. Fu, M. Shimura (Eds.), Fuzzy Sets and their Applications to Cognitive and Decision Processes. Academic Press, New York

Sameena K, Sunitha MS(2006) Strong arcs and maximum spanning trees in fuzzy graphs. Int J Math Sci 5:17-20

Tan YJ(2013) On transitivity of generalized fuzzy matrices. Fuzzy Sets and Syst 210: 69-88

Viedma E H, Herrera F, Chiclana F, Luque M(2004) Some issues on consistency of fuzzy preference relation. Eur J Oper Res 154:98-109

Xu YJ, Patnayakuni R, Wang HM(2013) The ordinal consistency of a fuzzy preference relation.Inf Sci 224:152-164

Xu YJ, Wang HM(2013) Eigenvector method, consistency test and inconsistency repairing for an incomplete fuzzy preference relation. Appl Math Model 37:5171-5183

Zadeh LA(1965) Fuzzy sets. Inf Cont 8:338-353

Zadeh LA(1971) Similarity relations and fuzzy orderings. Inf Sci: 177-200

Zhang HM(2019) Revisiting multiplicative consistency of interval fuzzy preference relation. Comput Ind Eng 132:325-332 
Zhang HP, Fernández RP, Baets BD(2020) Fuzzy betweenness relations and their connection with fuzzy order relations. Fuzzy Sets and Syst 384:1-22 
Figures

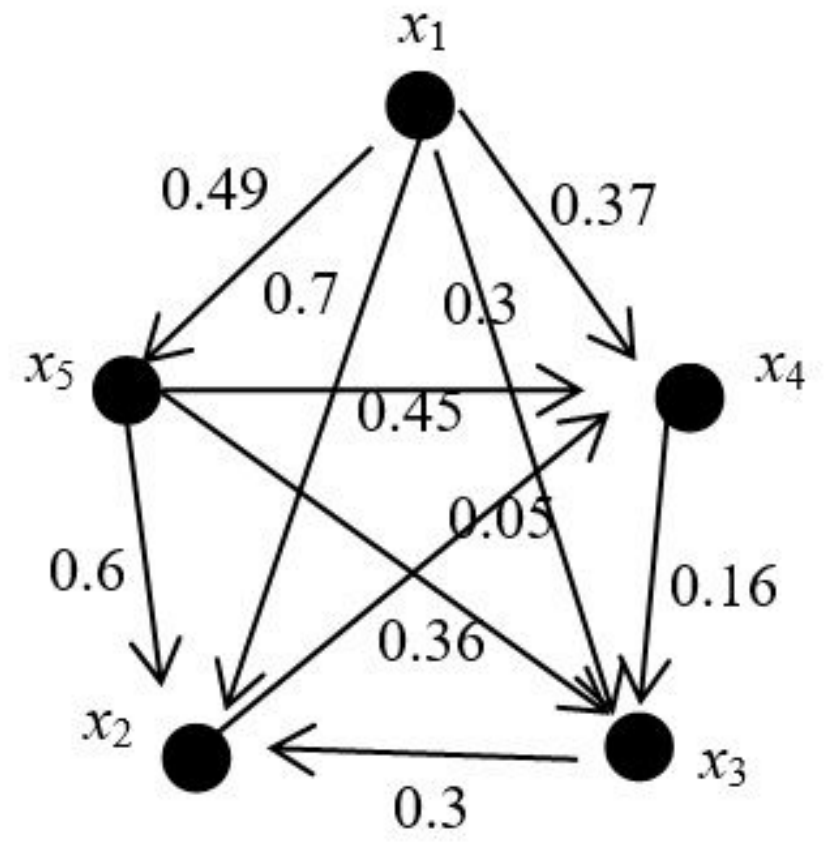

Figure 1

Graph of R of Example 1 